\title{
Neural Analysis of Mobile Radio Access Network
}

\author{
Kimmo Raivio \\ Helsinki University of Technology \\ Laboratory of Computer and Information Science \\ P.O. Box 5400, FIN-02015 HUT, Finland \\ Kimmo.Raivio@hut.fi
}

\author{
Olli Simula \\ Helsinki University of Technology \\ Laboratory of Computer and Information Science \\ P.O. Box 5400, FIN-02015 HUT, Finland
}

\author{
Jaana Laiho \\ Nokia Networks \\ P.O.Box 301, FIN-00045 Nokia Group \\ Finland
}

\begin{abstract}
The Self-Organizing Map (SOM) is an efficient tool for visualization and clustering of multidimensional data. It transforms the input vectors on two-dimensional grid of prototype vectors and orders them. The ordered prototype vectors are easier to visualize and explore than the original data. Mobile networks produce a huge amount of spatiotemporal data. The data consists of parameters of base stations (BS) and quality information of calls. There are two alternatives in starting the data analysis. We can build either a general one-cell-model trained using state vectors from all cells, or a model of the network using state vectors with parameters from all mobile cells. In both methods, further analysis is needed to understand the reasons for various operational states of the entire network.
\end{abstract}

\section{Introduction}

Data mining and exploration is an expanding new area of research in artificial intelligence and information management. The objective of data mining is to extract relevant information from databases containing large amounts of information. Typical data mining and analysis tasks include classification, regression, and clustering of data, determining parameter dependencies, and finding various anomalies from the data. In many engineering applications, the dimension of complex data is too large for human observation. Therefore, extracting relevant information from the data calls for intelligent and adaptive computational methods. Artificial Neural Networks (ANNs) have successfully been used in various intelligent data engineering applications.
The Self-Organizing Map (SOM) is a widely used neural network algorithm [8]. It has several beneficial features making it a useful tool in data mining and exploration. The SOM forms a nonlinear, topology-preserving mapping from the input to the output space. The SOM follows the probability density function of the underlying data and functions, thus, as an efficient clustering and data reduction algorithm. The SOM is readily explainable, simple and - perhaps most importantly - highly visual. SOM based methods have been applied in the analysis of process data, e.g., in steel and forest industry [9]. In addition, the SOM has been used in analysis and monitoring of telecommunications systems. Applications include novel equalizer structures for discrete-signal detection and adaptive resource allocation in telecommunications networks. In the current research, the goal is to develop efficient adaptive methods for monitoring the mobile network behavior and performance. Special interest is on finding clusters of mobile cells, which can be configured using similar parameters.

The SOM algorithm is able to perform both data clustering and visualization. The benefit of using SOM is in visualization of interesting parts of data. The algorithm moves the nodes of the map towards the areas of higher density of mapped input vectors. As a result, the SOM efficiently visualizes the clusters. In this work, a batch variate of the original SOM algorithm is used. The samples collected from a fixed time interval are first averaged over the topological neighborhoods of the respective winner cells in the map. After that the node vectors are updated in one step using these averaged values, as in the classical K-means algorithm [10].

The data used in this work has been generated using wideband code division multiple access (WCDMA) radio network simulator [7]. The WCDMA radio networks used 
in this study have been planned to provide 64-kbps service with $95 \%$ coverage probability, and with reasonable $(2 \%)$ blocking. The microcellular scenario depicted in Fig. 1 is capable of providing 2 to 3 fold (depending on service mix) the capacity of the macrocellular solution. The propagation model in the macrocellular case has been standard Okumura-Hata [1] [4] [11], in the microcellular case ray tracing has been utilized [6] [12].

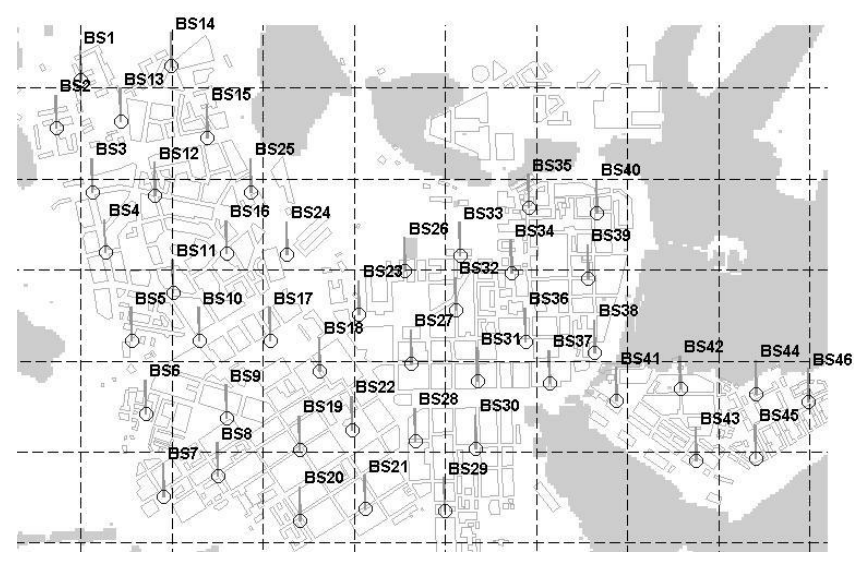

Figure 1. Helsinki city area with microcell scenario. The map area is approximately $8.6 \mathrm{~km}^{2}$.

The network configurations studied in this paper were macrocellular with 32 cells (11 base station sites) and microcellular with 46 omnidirectional cells. The macrocellular sites were sectored and equipped with 65-degree antennas, except one site was equipped with omnidirectional antenna. The network configurations were planned to cover the same area in Helsinki City. During the simulation the users of the network were circuit-switched, with 64-kbps, and the admission control was parameterized so that uplink interference had no impact on the admission process. Macrocell scenario is used to test the applicability of SOM clustering in general, and furthermore, to see whether same clustering techniques can be applied to different cell types.

The state of the network is characterized by 17 parameters of each base station. These parameters are stored every $100 \mathrm{~ms}$. The parameters include the number of users connected to BS, uplink noise raise in $\mathrm{dBs}$, average frame error rate (FER) and the real number of users, which are used in this study. During a call each mobile user keeps a list of possible BSs. Here, the maximum length of list is three. The user is connected to all of them, but uses only one of them at a time for the call. For the rest of the BSs on the list the user is in softhandover. So, the first number of users variable (noOfUsers) includes users in softhandover. A logarithmic scale with $10^{-2}$ as minimum is used for the error rate.

\section{Classification of cells using class frequen- cies}

Here, a method for classification of mobile cells will be presented. Preliminary results are also included. The method utilizes the SOM algorithm twice. A block diagram of the method is shown in Fig. 2.

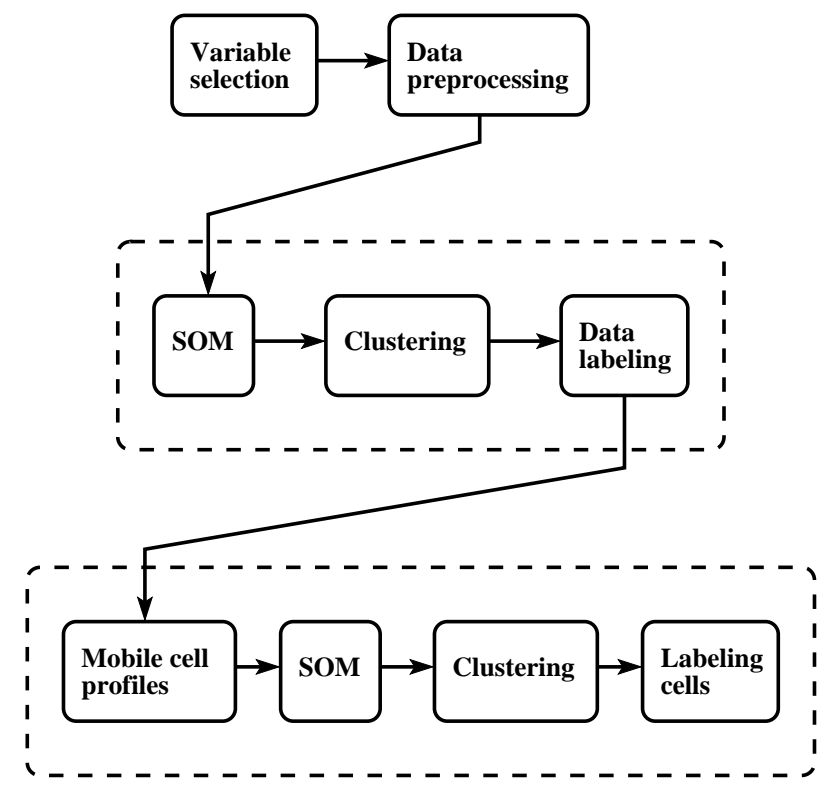

Figure 2. Classification of mobile cells using a general one cell model.

Analysis of the network starts with selection of variables and data preprocessing. The method is designed to handle any number of variables, but it gives more benefit if the number of variables is large. After preprocessing, the algorithm consists of two main steps.

At first, a general model of one mobile cell is built using the SOM. The model is an average of all used cells. Clusters of the SOM node vectors can be found manually using U-matrix presentation [14] of the vectors or some other clustering method can be used.

The second step of the algorithm builds a model of the network using cluster histograms of the data as profiles of mobile cells. Histograms for each mobile cell can be computed using the clusters as bins. Class frequencies are the data, which is used to train the second SOM and to find the best-matching units (BMU) of each cell.

\subsection{General mobile cell model}

In Fig. 3a the SOM component planes of the general mobile cell model of microcell network scenario are shown. 

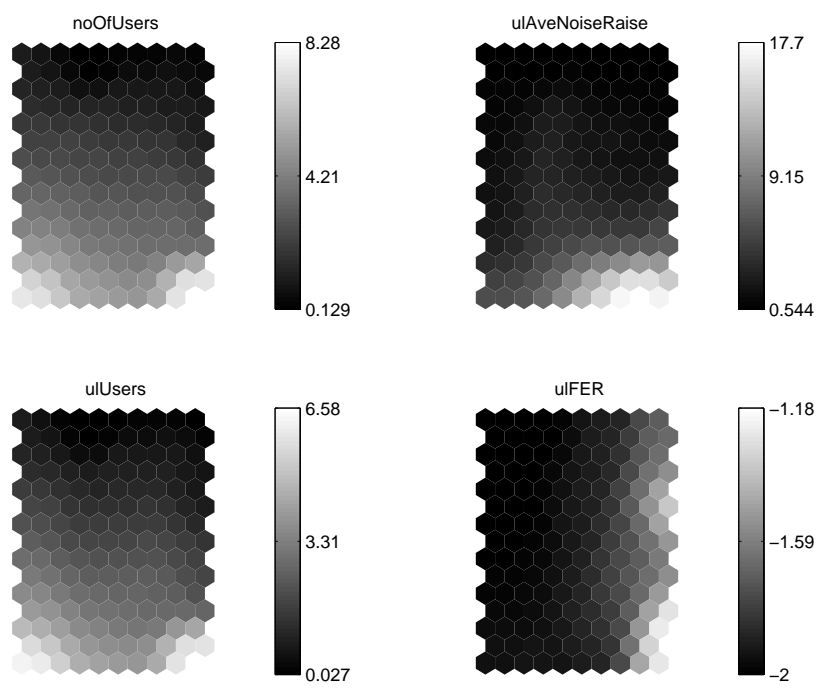

(a)

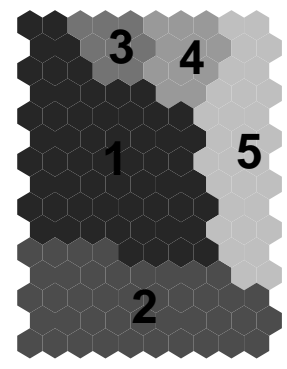

(b)

Figure 3. The component planes of SOM with denormalized colorbars (a) and five clusters of SOM node vectors given by Ward clustering (b).

The topology of the map is 2D $10 \times 15$ hexagonal grid. Clusters of the general model are searched using hierarchical clustering methods [3]. Hierarchical clustering techniques are either agglomerative, where at each round two smaller groups of samples are added together to form larger groups, or divisive, where the data vectors are separated in finer groupings.

In this paper, five agglomerative clustering techniques are used to find out the clusters of SOM codebook. The tested methods are Ward, centroid, and single, average and complete linkage clustering. Hierarchical clustering of SOM codebook has been considered in [16] and [13]. The computational complexity of hierarchical clustering of SOM codebook vectors is much lower than clustering directly the data.

The search for clusters of SOM node vectors is started by finding local minimas of the codebook. Different sets of local minimas are found depending on how large area around each node is verified until a node is decided to be local minima. The search area is limited by the maximum topological distance or the radius of a neuron. The distance to the nearest neuron equals one.

The local minimas are selected as the first centroids of the base clusters. The rest of the neurons are added to the base clusters using the selected clustering method. A Davies-Bouldin (DB) validity index [2] is computed for each clustering. For each clustering method the optimal number of clusters minimizes

$$
\frac{1}{C} \sum_{k=1}^{C} \max _{l \neq k}\left\{\frac{S_{c}\left(Q_{k}\right)+S_{c}\left(Q_{l}\right)}{d_{c e}\left(Q_{k}, Q_{l}\right)}\right\}
$$

where $C$ is the number of clusters, $S_{c}$ within-cluster distance and $d_{c e}$ between clusters distance, $Q_{k}$ and $Q_{l}$ are the clusters. The studied clustering methods differ in the definition of between clusters distance.

Table 1. Optimal base clusters of the first level SOM. Radius of local minima search defines the base clusters and the one corresponding the lowest DB index is selected. The best methods are marked with *.

\begin{tabular}{|l||c|c|c|}
\hline \multicolumn{4}{|l|}{ Microcell scenario } \\
\hline \hline Method & Radius & DB & C \\
\hline \hline Ward & 2.7 & 0.99 & $5^{*}$ \\
\hline centroid & 2.7 & 1.00 & 5 \\
\hline complete & 3.1 & 1.49 & 3 \\
\hline single & 3.1 & 1.11 & 3 \\
\hline average & 3.1 & 1.17 & 3 \\
\hline \hline Macrocell scenario & & \\
\hline \hline Method & Radius & DB & C \\
\hline \hline Ward & 3.7 & 1.01 & 2 \\
\hline centroid & 3.7 & 0.99 & 2 \\
\hline complete & 3.7 & 0.75 & $2 *$ \\
\hline single & 3.7 & 0.78 & 2 \\
\hline average & 3.7 & 0.84 & 2 \\
\hline \hline
\end{tabular}

In Table 1 the results of the search for the best base clusters are shown. The base clusters are found by quantizing the SOM codebook vectors to the local minimas of the codebook. Different base cluster sets are found using different radius for the search of local minimas. The radii giving the clustering with lowest DB indexes are shown. Because the studied clustering techniques are hierarchical, optimal clustering can be found by testing the clusterings found from the dendrograms against the validity index. However, 
this is not done at this level, because it is desirable for further studies to have more states. There are small variations in the the performance of the methods, but only complete linkage method has some difficulties in finding reasonable base clusters from microcell scenario data. For the microcell scenario Ward clustering gives the best results with lowest values of Davies-Bouldin index. Five base clusters or states of mobile cells minimize the validity index. The clustering result is shown in Fig. $3 \mathrm{~b}$ where the state 2 represents the higher load state, state 5 higher FER state and the others normal state.

\subsection{SOM of class frequencies}

The BMUs of data vectors give the state or the class of the cell. From a sequence of states we can compute the class frequencies of mobile cells. Using these frequency vectors as data to a second level SOM we get a SOM of frequency vectors or class histograms. The topology of the new SOM is $2 \mathrm{D}$ rectangular grid. Grid of size $9 \times 9$ nodes has been used for microcell scenario and grid of size $8 \times 8$ nodes has been used for macrocell scenario. The BMU search and the training of the map use Kullback-Leibler distance [5]. The Kullback-Leibler distance or relative entropy between two probability distributions $p_{X}(x)$ and $q_{X}(x)$ is defined by

$$
D_{p \| \mid q}=\sum_{x \in \mathcal{X}} p_{X}(x) \log \left(\frac{p_{X}(x)}{q_{X}(x)}\right)
$$

where the sum is over all states of the system (i.e., the alphabet $\mathcal{X}$ of the discrete random variable $X$ ). In Fig. 4 the second level SOM of histograms has been shown.

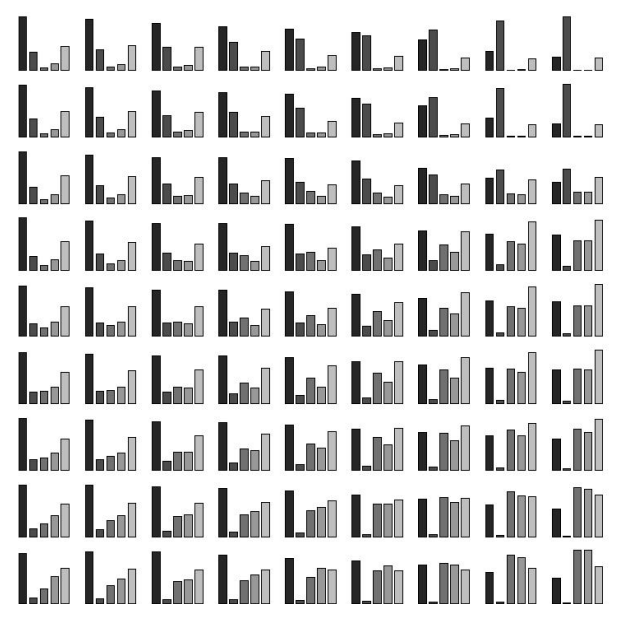

Figure 4. 9 × 9 SOM of class frequency histograms.
The same hierarchical clustering methods as in the first level SOM have been used to find clusters of the second level SOM except now the optimal clustering is also searched from dendrograms. From the results of Table 2 it can be seen that Ward clustering gives the best base clusters of mobile cells in microcell scenario. For both scenarios clustering with lower DB index can be found from the dendrograms as can be seen from Table 3. The dendrograms built on optimal base clusters and on first guess base clusters (radius equals one) are searched. For microcell scenario centroid and single linkage clustering give the lowest values of the validity index. All methods give two or three clusters. The results are usually quite similar, but sometimes a method may fail to find reasonable clustering. So, more than one method should always be used.

Table 2. The best base clusters for the SOM of histograms. Radius of local minima search defines the base clusters and the one corresponding the lowest DB index is the best (marked with *).

\begin{tabular}{|l||c|c|c|}
\hline \multicolumn{4}{|l|}{ Microcell scenario } \\
\hline \hline Method & Radius & DB & C \\
\hline \hline Ward & 4.1 & 0.73 & $2^{*}$ \\
\hline centroid & 4.1 & 0.73 & 2 \\
\hline complete & 4.1 & 0.73 & 2 \\
\hline single & 4.1 & 0.74 & 2 \\
\hline average & 4.1 & 0.73 & 2 \\
\hline \hline Macrocell scenario \\
\hline \hline Method & Radius & DB & C \\
\hline \hline Ward & 1.0 & 4.19 & 5 \\
\hline centroid & 1.0 & 4.19 & 5 \\
\hline complete & 3.1 & 0.73 & $4 *$ \\
\hline single & 3.1 & 0.73 & 4 \\
\hline average & 3.1 & 0.73 & 4 \\
\hline \hline
\end{tabular}

The clusters given by centroid algorithm and the BMUs of mobile cells are shown in Fig. 5. The BMUs of the original data have been printed using subscript 1 and the BMUs of the new data set with subscript $2\left(\mathrm{c} 44_{1}\right.$ means cell 44 with original data). In the original data set the admission control was turned off, which means that all users have access to the network all the time. Thus, the probability for lower call qualities should have been larger. In the new data set of the microcell network scenario, the admission control was turned on. Old first level clusters (Fig. 3b) were used to label the new data and compute new histograms.

The method divides the cells in two classes. By comparing Fig. 5 with Fig. 4 and Fig. 3b, we can see that the cells in lower right corner are characterized by higher FER and 
Table 3. Optimal clusters of the SOM of histograms given by dendrogram search. Radius for local minima search defines the base clusters for each method. The best methods are marked with *.

\begin{tabular}{|l|c||l|l|}
\hline \multicolumn{1}{|l|}{ Microcell scenario } \\
\hline \hline Method & Radius & DB & C \\
\hline \hline Ward & 1.0 & 0.90 & 2 \\
& 4.1 & 0.73 & 2 \\
\hline centroid & 1.0 & 0.31 & $2^{*}$ \\
& 4.1 & 0.73 & 2 \\
\hline complete & 1.0 & 0.63 & 2 \\
& 4.1 & 0.73 & 2 \\
\hline single & 1.0 & 0.31 & 2 \\
& 4.1 & 0.74 & 2 \\
\hline average & 1.0 & 0.73 & 3 \\
& 4.1 & 0.73 & 2 \\
\hline \hline Macrocell scenario & \multicolumn{2}{|l|}{} \\
\hline \hline Method & Radius & DB & C \\
\hline \hline Ward & 1.0 & 0.31 & $2^{*}$ \\
\hline centroid & 1.0 & 0.39 & 2 \\
\hline complete & 1.0 & 0.50 & 3 \\
& 3.1 & 0.49 & 3 \\
\hline single & 1.0 & 0.32 & 2 \\
& 3.1 & 0.31 & 2 \\
\hline average & 1.0 & 0.31 & 2 \\
& 3.1 & 0.31 & 2 \\
\hline \hline
\end{tabular}

lower number of users. All the cells at this location suffer from same problems.

Such a clustering is beneficial in the radio network optimization process. It is reasonable to assume that the configuration parameters for cells in one cluster within one optimized radio resource management function (admission control, handover control, etc.) are the same. Furthermore, on radio network "performance map" cells having close topographical location suffer from the same problem and can be, with high probability, fixed with the same solution.

The method described above classifies cells using class frequencies as models of cell behavior. The distributions describe how much a particular cell differs from a general cell model, which has been built using as much data as possible. The data which is used to build the lower level SOM in this method should be selected carefully so that it represents well all the possible states of the cells. If it does not, the lower level SOM should be trained again using new set of data. When SOM and hierarchical clustering are used twice on the same algorithm, it is not so clear how the first clustering should be done to obtain reasonable results on the

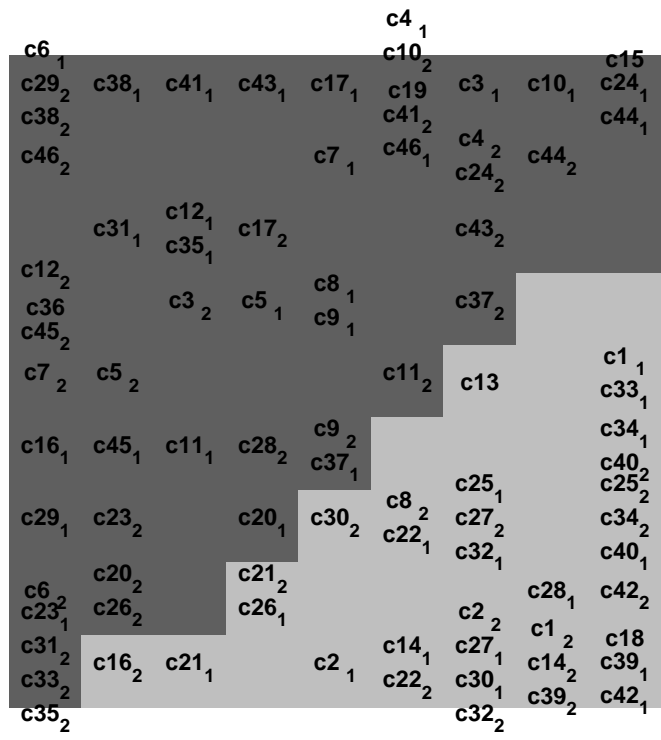

Figure 5. Clusters of mobile cells are found using class frequencies of the SOM, which is trained by number of connected users, uplink noise raise, real number of users and FER of each cell.

final level.

\section{Classification of cells using correlations of SOM component planes}

In this section, another method for clustering mobile cells on the basis of covariance matrixes of SOM component planes is presented. Also this method uses two level SOMs. In the previous method, the data was used to build a model of one base station. The same data can also be used to build models of the network. A block diagram of the method is shown in Fig. 6.

At first SOMs of one variable are built. Each of these SOMs is a model of the network. Next, the component planes of the SOMs are processed. In this paper, the covariance matrixes of the component planes are computed. Covariances of one or more variables are concatenated to be used as profiles of mobile cells. These profiles are the data to a second level SOM. The outputs of the second SOM are the clusters of mobile cells.

\subsection{SOM of one variable}

Data of each cell is masked so that one variable of each mobile cell is analyzed with the corresponding ones of the other cells. The data to be analyzed has been normalized 


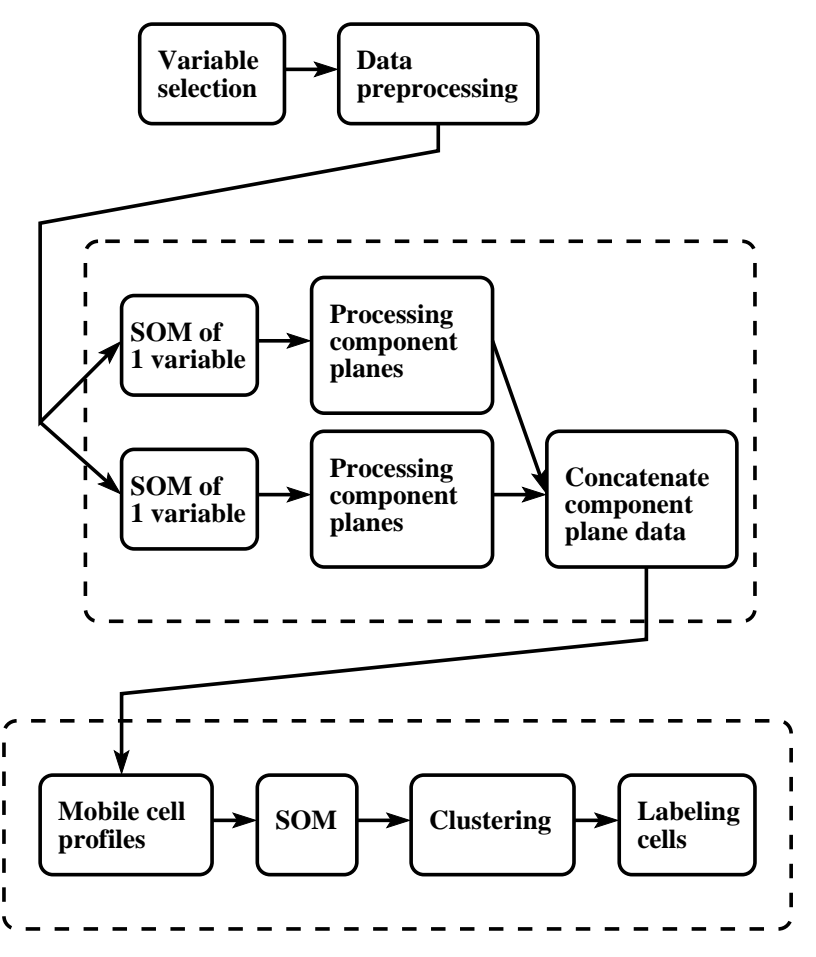

Figure 6. Classification of mobile cells using one variable SOMs as models of the network.

to zero mean and unit variance as one data vector over all the cells. Here the number of connected users, uplink noise raise, real number of users and uplink FER of each cell have been analyzed using the SOM. Hexagonal 2D neighborhood grid of $10 \times 15$ nodes is used. Fig. 7 shows the SOM component planes when the FER is studied. There is one component plane per each mobile cell. The parameter values of the network state at one moment can be read from similar locations on component planes. For example, upper left corner gives one possible combination of network error rates.

The component planes are visualized using a common color axis. This makes it possible to see the real error rates, but it also hides the smaller variations inside the cells. In the figure only some of the cells seem to differ from the common behavior. Cells 15, 24 and 44 have a lot higher FER than all the others.

\subsection{Correlation of SOM component planes}

If we are interested in, for example, to find out which mobile cells have similar FER distribution, the task of human analyzer can be made easier by further processing the component planes of SOM [15]. This kind of postprocessing is more important if the number of component planes is high.

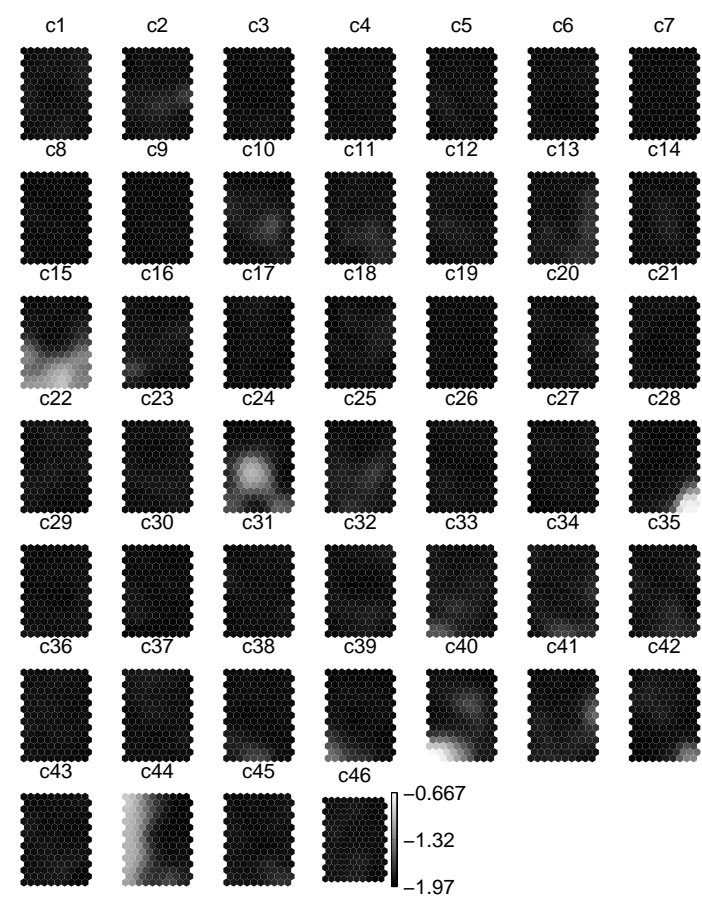

Figure 7. SOM component planes of the FERs. Minimum FER is fixed to $10^{-2}$.

The component planes are considered as separate figures. Covariance matrix of the figures is computed by first converting the figure dot values $c_{i j}^{n}$ to vectors $a^{n}$, where $i$ and $j$ are the coordinates on the map and $n$ is the mobile cell number. The length of each vector $a^{n}$ is the product of the dimensions of SOM topology.

The covariance matrix $C$ of the planes $a^{n}$ is the new data, which will be used on later studies. This data has one row for each mobile cell. A new second level SOM is trained using the covariance matrix. The topology of the new SOM is $2 \mathrm{D}$ rectangular grid. When 46 component planes are analyzed grid of size $9 \times 9$ nodes is used. For macrocell scenario network map of size $8 \times 8$ nodes is used. The covariance matrix row of each cell is mapped on the second level SOM and the BMU for each mobile cell is found. The map nodes are labeled using the results of BMU search. The SOM component planes have been reordered so that the similar ones locate near each other.

\subsection{Classification using several variables}

Several SOMs for different variables can be built and reorganized using the methods of Sections 3.1 and 3.2. The covariance matrixes $C_{k}$ of all first level SOMs can be combined so that we get a new data matrix $C=\left[C_{1} \ldots C_{k}\right]$, 
Table 4. Optimal base clusters of the second level SOM. Radius of local minima search defines the base clusters and the on corresponding to the lowest DB index is the best. The best methods are marked with *.

\begin{tabular}{|l||c|c|l|}
\hline \multicolumn{4}{|l|}{ Microcell scenario } \\
\hline \hline Method & Radius & DB & C \\
\hline \hline Ward & 1.0 & 0.94 & $8^{*}$ \\
\hline centroid & 1.0 & 0.95 & 8 \\
\hline complete & 1.0 & 1.16 & 8 \\
\hline single & 1.6 & 1.21 & 6 \\
\hline average & 1.6 & 1.14 & 6 \\
\hline \hline Macrocell scenario \\
\hline \hline Method & Radius & DB & C \\
\hline \hline Ward & 3.7 & 0.95 & 3 \\
\hline centroid & 3.7 & 0.92 & 3 \\
\hline complete & 3.7 & 0.89 & 3 \\
\hline single & 3.7 & 0.81 & $3^{*}$ \\
\hline average & 3.7 & 0.89 & 3 \\
\hline \hline
\end{tabular}

$k=1,2, \ldots$. Matrix $C$ has a row $C^{n}$ for each cell $n$. The row is a concatenated vector of cell correlations of used variables.

When the SOM is trained using this new data, the cells appear in a new order. The second level SOM can now be analyzed using the same hierarchical clustering methods as in Section 2 to find clusters of mobile cells. Now, Euclidean distance measure has been used. The base clusters are found by quantization of SOM codebook vectors to the local minimas of the codebook. Several sets of base clusters are found using different radius for local minima search. The radii giving best base clusters are shown in Table 4. The final clustering results are shown in Table 5. Ward and single linkage clustering give the best base clusters, but the DB indexes of all studied methods are about the same. However, for microcells scenario better clusterings can be found by analyzing the dendrograms. The clusters of the microcell scenario and the BMUs of the original data are shown in Fig. 8. Cells 15, 24 and 44 form one cluster and the rest of the cells form another cluster.

If new data is analyzed from a new cell or from a cell which has been analyzed before component plane representation of this data has to be constructed. The easiest way to do this is training the SOM again. When the SOM is trained the new data can be used in the BMU search or it can be masked out. If the new data is masked out in the BMU search, but used when the neurons are updated we can obtain similar SOM as before, but in addition we get the component planes for the new data. From the compo-
Table 5. Optimal clusters of SOM given by a search through dendrograms. Radius defines the base clusters for each method. The best methods are marked with *.

\begin{tabular}{|l|c||c|l|}
\hline \multicolumn{4}{|l||}{ Microcell scenario } \\
\hline \hline Method & Radius & DB & C \\
\hline \hline Ward & 1.0 & 0.65 & 3 \\
\hline centroid & 1.0 & 0.53 & 2 \\
\hline complete & 1.0 & 0.72 & 3 \\
\hline single & 1.0 & 0.40 & $2^{*}$ \\
& 1.6 & 0.40 & 2 \\
\hline average & 1.0 & 0.40 & 2 \\
& 1.6 & 0.40 & 2 \\
\hline \hline Macrocell scenario \\
\hline \hline Method & Radius & DB & C \\
\hline \hline Ward & 1.0 & 0.90 & 2 \\
& 3.7 & 0.95 & 3 \\
\hline centroid & 1.0 & 0.89 & 3 \\
& 3.7 & 0.92 & 3 \\
\hline complete & 1.0 & 0.90 & 2 \\
& 3.7 & 0.89 & 3 \\
\hline single & 1.0 & 0.90 & 2 \\
& 3.7 & 0.81 & $3 *$ \\
\hline average & 1.0 & 0.90 & 2 \\
& 3.7 & 0.89 & 3 \\
\hline \hline
\end{tabular}

nent planes new covariance matrices can be computed, new clusters can be found and the BMUs of the new and the old data can be found.

The method described above classifies mobile cells on basis of correlations of selected variables. A model of mobile network which describes the relations between mobile cells has been built.

\section{Conclusion}

In this paper, two new methods to find clusters of mobile cells in two radio access network scenarios have been presented. In the first method, a lower level SOM, which represents general mobile cell model is built. Histograms of the states of the base stations are built using clusters of lower level SOM. The same clusters can be used later to find out histograms of new data. Thus, the operational mode of each cell and the whole network can be monitored. In the second method, lower level SOMs of one variable are first build. Covariance matrices of the component planes of these SOMs are then used to train another map, which reorders the mobile cells.

States of one mobile cell and groups of similar mobile 


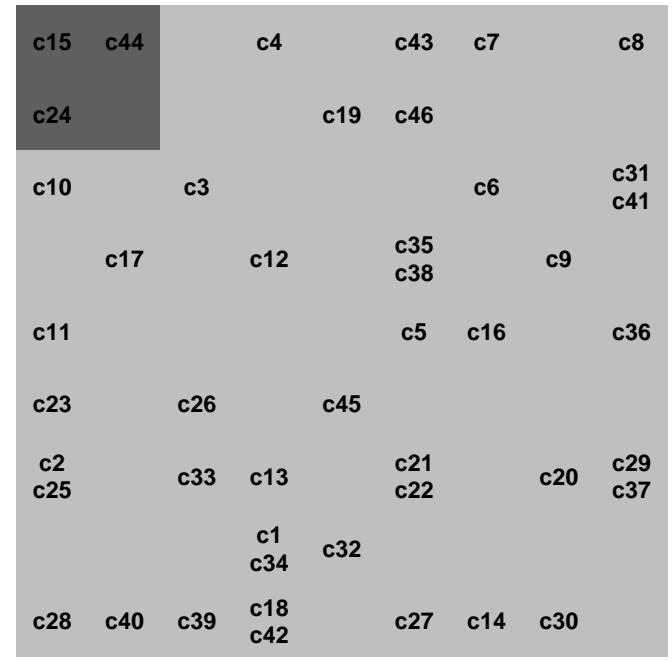

Figure 8. Clusters of mobile cells are found using correlations of all four one variable SOM component planes.

cells are found using agglomerative hierarchical clustering techniques on SOM codebook vectors. Usually, all the tested methods gave reasonable clusters with about the same validity index, but sometimes some method may fail to find clusters. Thus, more than one method should always be tested. However, a search through dendrograms built using both the first guess and optimal base clusters gave often better clusters when tested against Davies-Bouldin index.

The performance of clustering methods vary depending on selected set of variables and performed preprocessing like definition of outliers. The algorithms process data on multiple successive levels. Thus, a minor lower level change like organization of the SOM on another way might affect on final clustering. Usually, these changes are small, but it is also possible that a clustering method fails due to lower level reconfiguration. The algorithms should be tested more using real data in several real networks to obtain more reliable results.

Furthermore, in this paper it has been demonstrated that the SOM provides powerful means to move from time consuming and ineffective per cell optimization to cell cluster optimization. It has also been shown that the same methods can be used with a network of any type of cells.

\section{Acknowledgment}

The study has been financed by Nokia Networks, Nokia Mobile Phones and the Technology Development Centre Finland (TEKES) which is gratefully acknowledged. Fur- thermore, the authors wish to thank Jukka Henriksson, Ari Hämäläinen and other colleagues from Nokia Research Center for valuable comments during the work.

\section{References}

[1] Urban transmission loss models for mobile radio in the 900 and $1800 \mathrm{MHz}$ bands, September 1991. COST 231, $\mathrm{TD}(91) 73$.

[2] D. L. Davies and D. W. Bouldin. A cluster separation measure. IEEE Transactions on Pattern Analysis and Machine Intelligence, 1(2):224-227, April 1979.

[3] B. S. Everitt. Cluster Analysis. Arnold, 1993.

[4] M. Hata. Empirical formula for propagation loss in land mobile radio services. IEEE Transactions on Vehicular Technology, 29(3):317-325, August 1980.

[5] S. Haykin. Neural Networks, a Comprehensive Foundation. Macmillan, 1999.

[6] K. Heiska and A. Kangas. Microcell propagation model for network planning. In Personal, Indoor and Mobile Radio Communications, volume 1, pages 148-152, 1996.

[7] S. Hämäläinen, H. Holma, and K. Sipilä. Advanced WCDMA radio network simulator. In Personal, Indoor and Mobile Radio Communications, volume 2, pages 951-955, Osaka, Japan, September 12-15 1999.

[8] T. Kohonen. Self-Organizing Maps. Springer-Verlag, Berlin, 1995.

[9] T. Kohonen, E. Oja, O. Simula, A. Visa, and J. Kangas. Engineering applications of the self-organizing map. Proceedings of the IEEE, 84(10):1358-1384, October 1996.

[10] Y. Linde, A. Buzo, and R. Gray. An algorithm for vector quantizer design. IEEE Transactions on Communications, 28(1):84-95, January 1980.

[11] Y. Okumura, E. Ohmori, T. Kawano, and K. Fukuda. Field strength and its variability in the VHF and UHF land mobile service. Review Electronic Communication Lab., 16(910):825-873, 1968.

[12] J. Rajala, K. Sipilä, and K. Heiska. Predicting in-building coverage for microcells and small macrocells. In IEEE Vehicular Technology Conference, volume 1, pages 180-184, 1999.

[13] M. Siponen, J. Vesanto, O. Simula, and P. Vasara. An approach to automated interpretation of SOM. In N. Allinson, H. Yin, L. Allinson, and J. Slack, editors, Advances in SelfOrganizing Maps, pages 89-94. Springer, 2001.

[14] A. Ultsch and H. P. Siemon. Kohonen's self organizing feature maps for exploratory data analysis. In Proceedings of the International Neural Network Conference, pages 305308, Dordrecht, Netherlands, 1990.

[15] J. Vesanto. SOM-based data visualization methods. Intelligent Data Analysis, 3(2):111-126, 1999.

[16] J. Vesanto and E. Alhoniemi. Clustering of the selforganizing map. IEEE Transactions on Neural Networks, 11(3):586-600, May 2000. 